\title{
Baudelaire au miroir de Malraux
}

Moncef Khémiri

(Magazine littéraire $n^{\circ}$ 54, 1971: 14.)

-(PICHOIS, Claude. "Malraux et Baudelaire". Europe ${ }^{\circ}$ 727-728, 1989: 167-177.)

(Idem: 170.)

(Cf. "Adresse aux intellectuels". CEuvres complètes. Paris: Gallimard, 1989.)

(CASTEX, P.-G. Baudelaire, critique d'art. Paris: SEDES, 1969, note liminaire.)
N'oubliez pas que jappartiens à cette génération qui a vu les Fleurs du Mal entrer dans le domaine public.

André Malraux

C'est à Claude Pichois que revient le mérite d'avoir ouvert la voie à l'étude comparée de la pensée esthétique de Malraux et de celle de Baudelaire. En 1989, il avait en effet publié dans le numéro spécial que la revue Europe avait consacré à André Malraux, un excellent article intitulé Malraux et Baudelaire* dans lequel il avait souligné, avec raison, la place importante qu'occupe l'auteur des Fleurs du mal et des Curiosités esthétiques dans la pensée d'André Malraux. "La fidélité de Malraux à Baudelaire", écrit-il, "resta constante, plus ou moins expressément affirmée, jusqu’à la fin de sa vie." En effet, Malraux, dans ses articles de critique littéraire comme dans ses essais esthétiques, se réfere souvent à Baudelaire. "Les mentions explicites, peu nombreuses de 1920 à 1937 - six jusqu'en 1934 - deviennent de plus en plus nombreuses à partir comme il est normal - de la publication de La Psychologie de l'art, dans Verve (décembre 1937)", écrit Claude Pichois.

L'admiration de Malraux allait aussi bien au grand poète qu'au critique d'art. On le voit en effet souvent citer des vers, sinon des strophes entières des "Phares", de "La chevelure" ou du "Balcon", mais il voue aussi une admiration sans bornes au critique d'art. Malraux tient en effet Baudelaire pour "le plus grand critique d'art de la France et sans doute de l'Europe". *

En 1967, pour commémorer le centenaire de la mort de Baudelaire, Malraux, ministre de la culture du général de Gaulle, décide d'organiser une grande exposition au Petit Palais, et demande à Maurice Sérullaz, d'insister sur l'importance du critique d'art. Mais en raison du caractère ambitieux de ce projet et des événements de mai 68, l'exposition sera retardée. Elle n'eut lieu qu'à la fin de l'année, exactement entre le 23 novembre 1968 et le 17 mars 1969. Cette exposition représenta un grand événement culturel: elle a procuré, écrit P. G. Castex, "aux fervents de Baudelaire, le plaisir de voir rassemblées, en grand nombre, des toiles, des gravures, des statues qu'il a commentées et jugées". * 
Je voudrais dans cet essai ajouter de nouveaux éléments au dossier Malraux / Baudelaire. Ces éléments proviennent principalement de trois sources: la correspondance de Malraux, sa "bibliothèque esthétique" et les dossiers de genèse de La Métamorphose des dieux que j'ai eu l'occasion de consulter entre 2000 et 2004, pour les besoins de l'édition des Ecrits sur l'art d'André Malraux en Pléiade.

Comme on le sait, Malraux avait souhaité, en 1967, pour la commémoration du centenaire de la mort de Baudelaire, ériger la statue du Faucheur de Picasso, agrandie à des dimensions monumentales, à la pointe de l'Ile Saint-Louis en hommage aux Fleurs $d u$ Mal. ${ }^{1}$ Beaucoup de critiques ont mis en doute l'existence de ce projet. Or la consultation de la correspondance de l'auteur nous apporte la confirmation qu'il a bel et bien existé et nous montre que Malraux avait fait part de ce projet à Picasso. En effet dans une lettre, datée du $1^{\text {er }}$ juillet 1967, Malraux écrit en effet au peintre:

\section{Monsieur Pablo Picasso}

à Mougins

[...]

Mais j'espère vous convaincre que le Faucheur, agrandi, ferait un admirable monument à Baudelaire...

Portez-vous bien, peignez beaucoup et croyez à mes amitiés.

\section{André Malraux*}

En 1974, sans s'arrêter sur les raisons qui ont empêché la réalisation de ce projet, Malraux a présenté dans l'essai qu'il a consacré à Picasso qui venait de mourir, une photographie de cette statuette du Faucheur, suivie de ces mots de l'écrivain: "Je voulais en faire le monument aux Fleurs du mal'.

Mais outre ce précieux témoignage que la correspondance nous apporte, la bibliothèque de l'auteur et ses manuscrits nous révèlent respectivement à quelles sources il a puisé et sous quelles formes sont apparues les premières évocations de Baudelaire dans l'Introduction générale de La Métamorphose des dieux. Ces données peuvent être extrêmement utiles pour qui aimerait connaître comment Malraux a lu et commenté la pensée esthétique de Baudelaire.

\footnotetext{
${ }^{1}$ Ce projet est évoqué dans Le Miroir des limbes. (MALRAUX, André. CEuvres complètes. Paris: Gallimard, 1996, t. III.: 709.) Voir aussi le débat qui a suivi les communications d'André Holleaux et d'André Bernard "Malraux Ministre", in André Malraux, l'homme des univers. Verrières-le-Buisson: Comité national André Malraux, 1989: 184.
}

"(MALRAUX, André. CEuvres complètes. Paris: Gallimard, 2004, t. IV et V.)

' (Référence de la lettre. Cote: MLX 877. Bibliothèque littéraire Jacques-Doucet, Paris.)

"(MALRAuX, André. La Tête d'obsidien. Paris: Gallimard, 1974: 34.) 
(MALRAUX, André. 2004, t. IV: 1081-1084.)

- (Bibliothèque André Malraux, Inventaire sommaire des publications sur l'art, Frontispice de Hans Hartung. Paris: Musée National d'art moderne, Centre Pompidou, 1986.)

(Paris: Club des Libraires de France, 1956.)

(BAUDELAIRE, Charles. Curiosités esthétiques. L'Art romantique. Paris: Garnier, 1986: 217 , note 1 )
Dans ce texte, $\mathrm{j}$ 'aborderai trois thèmes qui me paraissent importants: "Malraux lecteur de Baudelaire", "Variation sur un thème: Baudelaire au Louvre" et enfin "Baudelaire ou l'émergence du fait pictural".

Je terminerai mon article par la présentation de l'un des quatre fragments inédits qui se rapportent à Baudelaire et qui ont été publiés par nos soins dans la récente édition des Ecrits sur l'art d'André Malraux dans la Bibliothèque de la Pléiade.

\section{Malraux lecteur de Baudelaire}

Après la mort d'André Malraux en novembre 1976, Madeleine Malraux, son épouse, et sa fille Florence Resnais, ont remis au Centre de Documentation du Musée National d'Art Moderne (Centre Georges Pompidou) les ouvrages sur l'art que comprenait sa bibliothèque. Ces ouvrages forment l'actuel "Fonds Malraux" au Musée National d'Art Moderne. En 1986, a été publié le catalogue: Bibliothèque André Malraux qui correspond à un inventaire de tous ces ouvrages que les chercheurs peuvent consulter à la Réserve du Centre de documentation du Musée. Ce fonds est constitué de deux mille vingt-quatre ouvrages publiés dont bon nombre sont annotés de la main même de Malraux. Parmi les nombreux ouvrages qui traitent de l'art et de la critique d'art au XIX siècle, on en trouve un qui est extrêmement important pour notre propos aujourd'hui à savoir l'édition par Bernard Gheebrant des écrits de Baudelaire sur l'art sous le titre: Baudelaire, critique d'art, Curiosités esthétiques, Poèmes, Euvres diverses, Lettres.

Le livre de Gheerbrant semble avoir intéressé Malraux au plus haut point. Il y a trouvé sûrement une confirmation de ses propres intuitions concernant la méthode du critique d'art, la nécessité du style pour l'artiste et enfin, et l'importance du "faire" pictural. En effet aux pages 30-31, Malraux souligne ce paragraphe extrait de "L'Exposition universelle de 1855" où Baudelaire écrit:

Il me serait trop facile de disserter subtilement sur la composition symétrique ou équilibrée, sur la pondération des tons, sur le ton chaud et le ton froid, etc. O vanité! je préfere parler au nom du sentiment, de la morale et du plaisir. [...] Il m’arrivera souvent d'apprécier un tableau uniquement par la somme d'idées ou de rêveries qu'il apportera dans mon esprit.

Cette "critique du sentiment au sens surnaturaliste du terme"* séduit Malraux qui est insatisfait de l'histoire de l'art qui réduit l'étude de l'œuvre d'art à un relevé de formes et de couleurs. 
Mais Malraux dépassera l'esthétique du "sentiment individuel" et de "la délectation" dont Baudelaire est encore tributaire, vers une métaphysique de l'art. Malraux semble avoir tout simplement pris Baudelaire au mot, car celui-ci avait déjà écrit dans le Salon de 1846: "la critique touche à chaque instant à la métaphysique."* Il n'en demeure cependant pas moins vrai que Baudelaire représente à ses yeux un précurseur dans la mesure où il a libéré l'œuvre d'art de l'histoire de l'art et de l'esthétique classique, et a fait une large place au sentiment individuel.

Le second passage souligné par Malraux concerne l'évocation par Baudelaire du tableau de Delacroix L'Entrée des Croisées à Constantinople:

Mais le tableau des Croisés est si profondément pénétrant, abstraction faite du sujet, par son harmonie orageuse et lugubre! Quel ciel et quelle mer! Tout y est tumultueux et tranquille, comme la suite d'un grand événement.

Dans le chapitre 4 de L'Intemporel, Malraux écrit à propos de Delacroix:

Il n'entreprend pas, lorsqu'il commence l'Entrée des Croisés, de représenter "ce qui s'est passé en 1204", mais de créer "par l'harmonie orageuse et lugubre" ${ }^{2}$ dont parle Baudelaire, le monde légendaire où les hampes des lances et les ailes déployées des casques se dressent sur la retombée des incendies.

Quant au troisième passage, il concerne le problème du style tel que Baudelaire l'aborde à propos d'Ingres dans le Salon de 1859:

Voilà sans doute, se dit-il, un curieux caractère, beauté ou grandeur, j'exprimerai cela soigneusement, je n'en omettrai rien, mais j'y ajouterai quelque chose qui est indispensable: le style. Et nous savons ce qu'il entend par le style [...], c'est une poésie étrangère, empruntée au passé.

Cette définition du style comme la poésie du passé trouve une résonance profonde dans l'imaginaire de Malraux, où la création moderne tire son originalité des relations qu'elle tisse avec les œuvres les plus éloignées dans l'espace et dans le temps, comme La Tête de mort de Picasso tire sa stridence de sa parenté avec le crâne d'obsidienne

\footnotetext{
2 "Mais le tableau des Croisés est si profondément pénétrant, abstraction faite du sujet, par son harmonie orageuse et lugubre! Quel ciel et quelle mer! Tout y est tumultueux et tranquille, comme la suite d'un grand événement." (BAUDELAIRE, Charles. "Exposition universelle de 1855 - Beaux-Arts. III Eugène Delacroix”. 1986: 233.)
}

* (L'Intemporel. Paris: Gallimard, 1976: 104.)

"(Idem: 145.) 
- (MALRAUX, André. L'Irréel. Paris: Gallimard, 1974: 51. Voir aussi MALRAUX, André. L'Intemporel. Paris: Gallimard, 1976: 167-168.)

(Op. cit.: 157.)

- (MALRAUX, André. Le Musée imaginaire. Genève: Albert Skira, 1947: 36.)

- (MALRAUX, André. Les Voix du silence. Paris: Gallimard, 1951: 114.)

(MALRAUX, André. 1974: 230.)

-(Idem: 231.)

•(Idem: 246.)

(Idem: 29.) aztèque. De même dans L'Irréel, Malraux n'a-t-il pas associé les personnages de Piero della Francesca à Arezzo, à "Limmobilité d'outretombe des pharaons". "Le style apparaît ainsi comme l'ombre du passé qui transfigure les formes que l'artiste invente.

Le quatrième passage est extrait de ce même Salon de 1846 où Baudelaire oppose l'œuvre finie à l'œuvre faite: “[...] Ensuite, [...] il y a une grande différence entre un morceau fait et un morceau fini, qu'en général, ce qui est fait n'est pas fini, et qu'une chose très finie peut n'être pas faite."** Dans Le Musée imaginaire, l'auteur rappelle cette distinction introduite par Baudelaire et l'étend à d'autres domaines artistiques:

D'où la découverte, que Baudelaire allait rappeler à propos de Corot, qu' “une œuvre faite n'était pas nécessairement finie, une œuvre finie pas nécessairement faite". L’art égyptien primitif, l'art assyrien, comme l'art roman se refusaient au fini autant que Corot [...].

Malraux qui accorde plus d'importance à la création qu’à la perfection $^{3}$ adopte cette distinction baudelairienne et l'exploite dans les Voix du silence où il oppose le "faire" au "rendu", et dans L'Irréel où il s'attache à souligner le goût du Tintoret et de Giorgione pour la matière picturale, qui se manifeste à travers la pratique de la "touche distincte" laissant voir "les marques de la main", de "l'exécution visible"* qui donne à l'oeuvre le charme de l'esquisse; ce qui conduit l'Arétin à déclarer qu'il préfere "les esquisses aux tableaux." Cette idée 4 reviendra dans les premières pages de L'Intemporel l'esquisse, l'œuvre inachevée est opposée au "tableau fini". *

Le cinquième passage qui a intéressé Malraux dans le Baudelaire critique d'art de Gheerbrant concerne Manet dont l'œuvre représente, à ses yeux, la véritable révolution picturale du XIXe siècle. Il souligne à la page 174 les commentaires de Gheebrant au sujet de l'Olympia:

3 “La Création m'a toujours intéressé plus que la perfection", écrit-il dans la préface des Chênes quion abat. (Paris: Gallimard, 1971: 7.)

${ }^{4}$ Dans le livre de Jacques Charpier et Pierre Seghers, L'Art de la peinture (Paris: Seghers, 1973.), Malraux semble avoir trouvé, dans les notes de Léonard de Vinci, une confirmation des propos de Baudelaire sur le faire pictural. Ainsi à page 166 de cette anthologie, il a coché à côté du passage où les auteurs citent Léonard de Vinci parlant de la nécessité de laisser inachevées les petites figures de tableau: "Tu te borneras $\mathrm{O}$ peintre, à esquisser tes petites figures sans les achever, sinon l'effet produit serait contraire à la nature, ta maitresse." Dans L'Intemporel, Malraux fera de Léonard le précurseur de l'œuvre inachevée qui tire toute sa qualité du faire pictural, de son inachèvement même. 
L'Olympia, nouveau scandale de 1865: la lumière devient forme. Un grand aplat de tons chauds, le corps nu est structuré par les tons froids des vêtements qui l'entourent. L'auteur n'exprime plus des passions comme Goya, une sublimation du désir comme Ingres, [...] il crée une réalité plus importante que la réalité intérieure.

Et Gheebrant de citer Malraux lui-même qui a écrit dans la Psychologie de l'art:

Le sujet doit disparaître parce qu'un nouveau sujet apparait qui va rejeter tous les autres: la présence dominatrice du peintre lui-même. Pour que Manet puisse peindre Clemenceau, il faut qu'il eût résolu d'oser y être tout, et Clemenceau rien.

Malraux a dû sans doute beaucoup apprécier que la pertinence de ses analyses ait été reconnue par les critiques et que ses textes soient cités à côté de ceux de Baudelaire. ${ }^{5}$

Baudelaire est d'ailleurs tellement présent dans la pensée de Malraux qu'il finit par devenir un personnage apparaissant dans une séquence narrative, ce qui est extrêmement intéressant dans cette méditation esthético-philosophique qu'est La Métamorphose des dieux.

Variation sur un thème: Baudelaire au Louvre

Dans "L'Introduction générale" de La Métamorphose des dieux, Malraux, pour mettre en valeur l'originalité de la conception ouverte que l'homme moderne se fait de l'art, situe cette conception par rapport à celle de Baudelaire. Il imagine alors Baudelaire en visite à notre Musée du Louvre actuel, et tente de rendre compte de sa réaction devant certaines oeuvres qui l'auraient beaucoup surpris soit parce qu'elles ont été découverte après sa mort, soit parce qu'ils ne les tenaient pas, à proprement parler, pour des oeuvres d'art. Dans cette visite imaginaire au Louvre des années cinquante, Baudelaire a comme guide son animal fétiche, un chat, qui est aussi rappelons-le, l'animal préféré de Malraux dont les funérailles dans la Cour Carrée du Louvre ont été d'ailleurs présidées par un chat égyptien:

\footnotetext{
${ }^{5}$ Jacques Charpier et Pierre Seghers ont présenté également dans L'Art de la peinture (Paris: Seghers, 1957), des extraits du Musée Imaginaire et des Voix du silence, p. 674-682. Pierre Daix, dans La Vie de peintre de Pablo Picasso (Paris: Seuil, 1977), cite également Malraux, p. 268.
} 
Imaginons, écrit Malraux, qu’un démon-gardien (en forme de chat), lorsque Baudelaire vient d'achever "Les Phares", ${ }^{6}$ lui dise: "Voyons un peu”, et l'introduise dans notre Louvre. / Baudelaire est d'abord stupéfait de la place prise par la sculpture - qu'il tient, comparée à la peinture, pour "un art des Caraïbes". ${ }^{8}$ Son propre Louvre est encore un musée d'antiques; ' au British Museum, les marbres du Parthénon font figure d'archaïques; la "Victoire de Samothrace" n'est pas encore découverte. Sans doute, il a parlé "d'une barbarie inévitable, synthétique, enfantine, qui reste souvent visible dans un art parfait (mexicaine, égyptienne ou ninivite) et qui dérive du besoin de voir les choses grandement". ${ }^{10}$

C'est en marge d'une étude sur Guys, et jamais il ne s'est référé avec quelque détail aux œuvres qui montrent cette barbarie; il célèbre seulement Michel-Ange et Puget. ${ }^{11}$ Que ni Viollet-le-Duc

"Si Malraux a choisi de citer "Les Phares", c'est parce que ce poème des Fleurs du mal donne une idée précise sur le musée imaginaire de Baudelaire. Les grands créateurs qui ont compté pour le poète sont d'ailleurs cités par l'auteur en note dans L'Intemporel, p.12, note 1.

${ }^{7} \mathrm{Au}$ Metropolitan Museum, le premier sujet d'étonnement de Baudelaire aurait été le nombre des œuvres: "Nous sommes portés à croire que son premier étonnement portera sur la multiplicité des œuvres. Or, s'il croyait qu'un siècle après sa mort, les chefs-d'œuvre seraient à peu près les mêmes, il supposait aussi que des œuvres de toute nature auraient émergé des civilisations." (Cf. "Le Problème fondamental du musée", La Revue des Arts, mars 1954: 4-6.)

${ }^{8}$ Ce propos figure en effet dans le Salon de 1846 où Baudelaire explique entre autres pourquoi il estime que la "sculpture est ennuyeuse", "brutale et positive comme la nature". Baudelaire écrit en effet: "L'origine de la sculpture se perd dans la nuit des temps, c'est donc un art des Caraïbes". Voir Charles Baudelaire, "Pourquoi la sculpture est ennuyeuse" in Salon 1948, Curiosités esthétiques et art Romantique. (Paris: Classique Garnier, 1962: 187.) Henri Lemaître explique la répulsion de Baudelaire devant les moyens de la sculpture par "l'intransigeance de son parti surnaturaliste". (Idem: 187, note 2).

9 “(...) les œuvres qu'il [Baudelaire] tenait pour les œuvres grecques capitales, ou bien ne sont pas grecques, ou bien sont remplacées dans notre admiration par des œuvres de beaucoup antérieures: l'art du musée de l'Acropole a remplacé 'l'antique" Cf. "Le Problème fondamental du musée" (Op. cit.). "Le Louvre n’est pas une galerie d'antiques développée: il est autre chose, et pose d'autres questions." (La Tête d'obsidienne: 104.)

${ }^{10}$ Cette citation de Baudelaire figure en effet dans la cinquième partie de l'étude consacrée à Constantin Guys sous le titre "Le peintre de la vie moderne", Curiosités esthétiques, L’art romantique. (Paris: Classiques Garnier, 1962: 469) Elle connaît de nombreuses occurrences dans les essais esthétiques de Malraux. Voir Le Musée imaginaire: 62, note 1, Les Voix du silence: 105, L'Irréel: 133, "Pour sauver les monuments de Haute-Egypte", in Oraisons funèbres, Euvres Complètes (Paris: Gallimard, 1996: 925, t. III).

11 "N'oublions pas que, pour Baudelaire, la sculpture, à l'exception de MichelAnge et de Puget qui appartiennent aux temps modernes, avait été 'un art des Caraïbes' et ne cessa jamais tout à fait de l'être." (MALRAUX, André. Le Mu- 
ni Victor Hugo ne nous égarent: les restaurations du premier suffisent à prouver que le gothique ${ }^{12}$ était alors une dépendance de l'archéologie, et l'art de sa sculpture, lettre morte. ${ }^{13}$ La résurrection de celle-ci, comme celle de l'égyptienne, en suit l'étude de près d'un siècle: jamais Baudelaire n'a cité Chartres. ${ }^{14}$

Dans les salles de peinture, il constate avec joie la disparition d'un italianisme et d'un académisme qu'il a dédaignés, la promotion de Delacroix, la gloire de Goya; mais lit-il sans surprise, sous une pietà découverte après sa mort à Villeneuve-lès-Avignon: "Ce tableau est parfois cité comme l'œuvre la plus importante de la

sée imaginaire. Paris: Gallimard, 1965: 146.) "Le Musée Imaginaire de Baudelaire: pas de sculpteurs avant Puget, sauf Michel-Ange, pas de primitifs. Les Phares commencent au XVI ${ }^{\text {ème }}$ siècle..." (La Tête d'obsidienne: 124).

${ }^{12}$ Voir aussi Les Voix du silence, p. 19. Dans le manuscrit de la préface, le développement consacré à l'attitude des romantiques envers les arts roman et gothique était assez important comme en témoigne le fragment composé de six feuillets, numérotés de $\mathrm{A}$ à $\mathrm{F}$, et qui commence à: "Nous savons ce que nous a apporté la peinture moderne[...]” jusqu’à: "[...] mais le Trésor du M. Imag., - empiriquement - sait assez bien ce qu'il appelle art”. (Méta Préface Manuscrit, carton 1/6, Dossier $\mathrm{n}^{\circ} 11$ ). Voir à ce propos "La genèse de quelques parties du texte" dans la notice du Surnaturel (Paris: Gallimard, 1977).

${ }^{13}$ Dans le discours qu'il a prononcé en 1954 au congrès d'Art et d'Archéologie réuni à New York, Malraux a mis en question le mythe médiéval du romantisme en ces termes: "Le romantisme a ressuscité un rêve du Moyen Age, des lieux historiques, un décor: il n'a jamais ressuscité un style. En Angleterre, comme en France, les restaurations ont détruit tout le génie qu'elles ont touché - en Angleterre, la quasi-totalité de la sculpture gothique. Le tympan de saint Denis, abbaye royale de France, est arraché au génie, vers le milieu du XIXe siècle, par un sculpteur qui 'modernise' le tympan des rois, auquel il ajoute sereinement son nom: Brun. Le musée ne connaît alors qu'une sculpture suprême: la sculpture antique, qu'il croit grecque." ("Le Problème fondamental du Musée" in La Revue numéro des Arts, mars 1954: 4) Malraux aurait pu citer aussi Michelet qui a dénoncé le gothique dans des termes particulièrement sévères. Voir Jules Michelet, "L'architecture rationnelle et mathématique - La déroute du gothique” (Paragraphe X de l'Introduction). Histoire de France, Tome troisième, Depuis la Renaissance jusqu'à la Ligue, Illustrée de 334 dessins. Paris: J. Hetzel et Cie, Editeurs, s.d., 1855: 26-30.

14 "Baudelaire qui cite Puget et Coustou, ne cite ni Chartres ni Moissac." Chemise gris-bleu, portant comme titre le numéro du chapitre "XVI". Maquette avec pages montées, imprimées et corrigées. Numérotées de 1 à 13 . Documents remis par Jacqueline Blanchard à M. Henri Godard. p. 1. “(...) Après la résurrection de cette peinture chrétienne, Baudelaire découvrirait celle de la sculpture chrétienne: j'ai dit qu'il ignorait la sculpture des cathédrales, qui joue pour nous le rôle que l'on sait." ("Le Problème fondamental du musée", op. cit.) Mais le plus souvent, c'est à Théophile Gautier que Malraux a fait le reproche d'avoir ignoré la cathédrale de Chartre: “Théophile Gautier, passant par Chartres, vers 1845, écrit: 'Je n'ai pas eu le temps de faire le détour pour voir la cathédrale.” (Idem: 4.) Voir aussi Le Musée imaginaire (Paris: Gallimard, 1965: 146.), et Le Miroir des limbes (CEuvres complètes, t. III: 745.) 
(Op. cit., planche $\left.\mathrm{n}^{\circ} 36.\right)$

(Op. cit.: 67.)

(Paris: Gallimard, 1952.)

(Op. cit., planche $\mathrm{n}^{\circ}$ 126.) (planche $n^{\circ} 160$ et 161. )

(Idem: 96, note 1.) peinture française"? Ni Giotto, ni Van Eyck ne figurent dans "Les Phares"... ${ }^{15}$ La place des tableaux chassés de son Louvre n'est pas occupée dans le nôtre par un éclectisme triomphant, comme on le prophétisait à l'envi; elle l'est par la prolifération presque exclusive des tableaux qu'on accrochait dans l'antichambre: les primitifs, qui s'accordent à la sculpture ressuscitée.

Et si, au sortir du vrai musée, le démon-gardien évoque le Musée Imaginaire, alors, depuis les mosaïques de Ravenne jusqu’à Grünewald, depuis les Rois de Chartres jusqu'aux figures océaniennes, notre temps apparait à Baudelaire comme celui de l'invasion du musée par un art qui n'était pas sa propre fin. ${ }^{16}$

Ce qui aurait donc surpris d'abord Baudelaire ce sont ces oeuvres qui étaient inconnues de lui comme "La Victoire de Samothrace", "entrée au Louvre un an après sa mort" précise Malraux dans "Le Problème fondamental du musée". En effet, "La Victoire de Samothrace", cette statue en marbre de l'époque hellénistique (exécutée vers 190 av. J.-C. et conservée actuellement au Louvre) qui a été découverte par le consul de France d'Andrinople en 1863 dans l'île égéenne de Samothrace, est entrée au Louvre en 1868, une année après la mort de Baudelaire. Allégorie de la Victoire, elle est représentée sous la forme d'une figure féminine aux ailes déployées, aujourd'hui acéphale et dépourvue de bras. Cette statue est fréquemment citée par Malraux qui en a présenté une reproduction photographique dans la plupart de ses essais (Le Musée imaginaire; "Les Voix du silence; 'Le Musée imaginaire de la sculpture mondiale, "La Statuaire; Le Musée imaginaire; ${ }^{*}$ et dans Le Surnaturel. ${ }^{*}$ Une longue note lui est réservée dans Le Surnaturel.) ${ }^{*}$ Elle est pour lui le plus bel exemple de l'œuvre d'art que le temps métamorphose

\footnotetext{
${ }^{15}$ Parlant de Baudelaire, Malraux écrit dans une version intermédiaire: "A peine citera-t-il incidemment, quelques peintres gothiques, en tant que précurseurs: Les Phares commencent au XVIème siècle" ("Malraux", Carton 1/6, Dossier n ${ }^{\circ} 4$, chapitre V, composé de 17 feuillets numérotés de 1 à 17, de: "Sans doute l'Europe (...)” jusqu’à: “(...) suscitées par le monde.”, p.13.).

16 “... Car on eût fort étonné ceux qui, jusqu’en 1850, refusaient l'hospitalité du Louvre aux [gothiques, biffé] [médiévaux, add.ms interl]- jugeant que leur place était toujours aux Monuments historiques - et même, peut-être, ceux qui l'exigeaient, si on leur avait affirmé, que cent ans plus tard, les chefs-d'œuvre gothiques [et les tableaux primitifs, add.ms interl.] seraient comparés aux plus grandes figures de Michel-Ange, et presque unanimement préférés à l'Apollon du Belvédère. Ce que vous appelez art, nous répondraient leurs ombres, n’est pas ce que nous appelions ainsi." Dossier 13, "Métamorphoses", chap. VI. $43 \mathrm{fts}$ dact \& mss ss nº. De: "Ainsi deux poussées singulièrement différentes...", jusqu’à: "Les romains accueillaient dans leur Panthéon, les dieux vaincus." $2^{\text {ème }} \mathrm{ft}$.
} 
en lui faisant subir des dégradations qui la révèlent à elle-même. Dans Les Voix du silence, Malraux écrit à son sujet:

En 1910, on croyait que la "Victoire de Samothrace", restaurée, retrouverait son or, ses bras et un buccin. Sans or, sans bras et sans buccin, elle a retrouvé sa proue, et trouvé le haut escalier du Louvre qu'elle domine comme un héraut du matin: ce n'est pas vers Alexandrie que nous la dressons, c'est vers l'Acropole. La métamorphose n'est pas un accident, elle est la loi même de la vie de l'œuvre d'art.

Il revient vers la fin de cet essai sur ce thème de la dégradation qui transfigure pour souligner la forme nécessaire et le caractère quasi fantastique qu'acquiert la statue: "Ce monstre radieux a perdu sa tête sans grand dommage, et n'eût pas perdu ses ailes sans mourir." Explicitant davantage la signification surnaturelle de cette figure, Malraux la présente dans Le Miroir des limbes, comme l'œuvre du destin:

... [l'action] de Victoire de Samothrace vient de ce que le destin a inventé le monstre parfait que les hommes ont cherché en vain dans les anges: les ailes étant les bras des oiseaux, la victoire n’est parfaite que sans bras.

Il en est de même de La Pietà d'Avignon (XVe. Siècle, Villeneuve-lès-Avignon) que l'on attribue à Enguerrand de Charonton ou Enguerrand Quarton (av. 1419-env.1466), qui est entrée au Louvre l'année de la mort de Cézanne, nous dit souvent Malraux, " c'est-àdire en 1906. Elle a été révélée au public "à l'exposition des Primitifs français en 1904”. Elle est avec le Boufécorché de Rembrandt, la Kermesse de Rubens et les Grandes danseuses vertes de Degas, l'une des œuvres qui a le plus marqué la sensibilité esthétique du jeune André Malraux.* On trouve dans la bibliothèque esthétique de Malraux l'ouvrage de Germain Bazin, La Pietà d'Avignon. XVIe siècle. . Baudelaire aurait été surpris de la place majeure que le Louvre moderne accorde à l'art médiéval auquel il a été, lui, complètement indifférent comme d'ailleurs bon nombre de ses contemporains, notamment Théophile Gautier, Delacroix et Hyppolite Taine.

Dans une version antérieure de ce passage, Malraux écrivait: "Car on eût fort étonné ceux qui, jusqu'en 1850, refusaient l'hospitalité au Louvre aux médiévaux - jugeant que leur place était toujours aux Monuments historiques - et même peut-être, ceux qui l'exigeaient, si on leur avait affirmé, que cent ans plus tard, les chefs-d'œuvre gothiques (et les tableaux 'primitifs') seraient com- 
• (La Tête d'obsidienne, op. cit.: 103-104.)

(Méta Préface Manuscit, Carton $1 / 6$, Dossier $\mathrm{n}^{\circ} 11$ Fragment composé de 8 feuillets numérotés de 94 à 100 commençant à: "Il s'agit ic d'une réflexion fondamentale $[\ldots]$ " jusqu'à: "[...] avec ses maîtres et ses disciples, ses prophètes et se scribes, ses fidèles et sa communauté, avec son", p. 99 bis100.) parés aux grandes figures de Michel-Ange, et presque préférés à l'Apollon du Belvédère.'

Cette visite imaginaire de Baudelaire au Louvre est un moment important dans la réflexion de Malraux sur ce qu'il appelle la métamorphose de l'oeuvre qui résulte aussi, et dans une large mesure, de la métamorphose de notre regard sur l'oeuvre.

Dans la conversation qu'il a eue avec Picasso en 1945, à Paris, dans l'atelier des Grands Augustins, Malraux rapporte avoir dit à l'artiste à peu près ceci: "Relisez Baudelaire, vous serez stupéfait de constater à quel point notre musée, en un siècle, est devenu différent du sien. J'essaie de faire l'inventaire./ D'abord, tout ce que nous avons découvert depuis 1900: Asie, Afrique, Amérique, précolombienne. Ce que nous avons ressuscité Byzance et l'art médiéval. Pour Baudelaire, la sculpture commence à Donatello.”*

Dans le manuscrit, nous trouvons deux variantes de cet épisode imaginaire dont Baudelaire est le héros. Dans la première, nous remarquons que ce n'est pas cette visite de Baudelaire au Louvre que l'auteur a d'abord imaginée, mais une autre scène montrant le poète examinant un livre d'art riche en reproductions photographiques: "Si Baudelaire avait feuilleté ces photographies, n'eût-il pas été surpris, plus que tout ce que j'ai dit, de l'intrusion de cette négation de la durée? Et (Mais biffé) ne l'eût-il pas étrangement retrouvée, s'il eût feuilleté un album de la peinture qui a succédé à celle (mot raturé illisible) de son temps? / Il aurait rencontré d'abord l'épisode impressionniste, et continuant de feuilleter, n'eût pu se méprendre à (sic) sa signification. / Il eût donc découvert d'un coup tout notre art, comme j'ai supposé plus haut que pourrait le découvrir une civilisation future. Ne l'eût-il pas jugé parent de tous ceux qu'il a ressuscités? Il savait que style ne veut pas dire stylisation, que hiératisme ne veut pas dire calligraphie; n'eûtil pas soupçonné dans le cubisme un parent de Byzance. / Encore eût-il ignoré ce que nous savons. Que tous les peintres modernes, qu'ils aient ou non la moindre conscience de lutter contre le temps, ont (suite de mots raturés) peint pour atteindre le pouvoir énigmatique par lequel les grands peintres de tous les temps étaient plus présents en eux que le monde qui les entourait? (...)”*

La seconde variante semble être davantage l'ébauche de la scène qui sera finalement retenue. L'auteur y écrit en effet: "J'imagine qu'un démon sous forme de chat à l'instant où Baudelaire vient d'écrire à Manet: '... mais vous n'êtes que le premier dans la 
décadence de votre art' lui dise: 'Voyons un peu' et lui permette de connaître ce que le Musée Imaginaire, cent ans plus tard aura retenu des ouvres qui, pour Baudelaire, appartiennent au passé. (Je serais fort curieux de connaître celui de 2060, même arrêté à Picasso...)" Le poète est d'abord stupéfait de la place prise par la sculpture. Celle-ci est, comparée à la peinture, un art des Caraïbes. (Suit un passage surchargé d'additions interlinéaires difficile à reconstituer: Elle [était biffé] avant tout l'antique; car du grand art grec, on ne connaissait guère que les statues du B. M. Lorsqu'il parle de [blanc], c'est en marge) car jamais, il ne se réfere aux œuvres auxquelles cette naïveté appartient (mot raturé illisible). Que Victor Hugo et Viollet-le-Duc ne nous égarent pas: les restaurations du second suffisent à prouver que l'art gothique appartenait alors à l'archéologie; les (un blanc) du premier qu'il appartenait au pittoresque (Suite de mots raturés et illisibles). Comme celles de l'art égyptien, sa découverte est du XIXe siècle, et sa résurrection du XXe [Quant à la peinture, Baudelaire (eût vu biffé) voit sans surprise la disparition d'un italianisme et d'un académisme et] (Cette dernière phrase est reprise en tête du $2^{\text {nd }}$ feuillet). Quant à la peinture, il constate sans surprise la disparition d'un italianisme et d'un académisme qu'il a dédaignés, la reconnaissance du talent de Delacroix, la gloire de Goya. (Mais biffé) Il eût été au moins intrigué (suite de mots raturés et illisibles) si la légende de la Pietà de Villeneuve avait porté: “Ce (cette toile biffé) tableau est tenu par beaucoup d'artistes vivants pour l'œuvre la plus importante de la peinture française"; et se fût aperçu que (suite de mots raturés et illisibles) le M.I. de la peinture s'était transformé de façon moins complexe, et beaucoup plus brutale qu'il ne l'eût supposé: il eût retrouvé ses maîtres en place, et les peintres qu'il avait dédaignés, chassés et remplacés en bloc par ceux qu'il avait appelés les primitifs. Et sans doute, des Rois de Chartres aux figures océaniennes, notre temps lui eût-il apparu comme celui de l'envahissement du musée par les œuvres des temps où l'idée d'art n'existait pas."

\section{Baudelaire ou l'émergence du fait pictural}

Bien que Malraux estime que le musée de Baudelaire est bien pauvre par rapport au nôtre ${ }^{17}$ et qu'il reproche au poète de n'avoir

\footnotetext{
17 "Baudelaire ne vit les œuvres capitales ni du Greco, ni de Michel-Ange ni de Masaccio, ni de Piero della Francesca, ni de Grünewald”. (Le Musée imaginaire, Op. cit.: 17; Le Surnaturel, Op. cit.: 2-3.)
} (Méta Préface Manuscrit,
Carton 1/6, Dossier $\mathrm{n}^{\circ} 11$.
Deux feuillets manuscrits
non numérotés.) 
- (Le Musée Imaginaire, op. cit.: 62.)

(Cf. la note 10 de cet article.)

( (L'Intemporel, op. cit.: 33.)

(Curiosités esthétiques. L'Art romantique, op. cit.: 117 .)

(L'Intemporel, op. cit.: 1415.) pas bien saisi l'importance des "arts précolombiens qui commençaient à sourdre” - N'en-a-t-il pas parlé comme d'une "barbarie [...] synthétique enfantine" dans ce qu'il avait écrit en marge d'une étude sur Constantin Guys?* - Malraux reconnaît, cependant, le rôle de précurseur que celui-ci a joué dans la promotion du fait pictural libéré de la fiction. Dans L'Intemporel, il met en valeur l'intérêt que Baudelaire a manifesté pour l'estampe chinoise. Il évoque comment le poète, fouillant les cartons du magasin "A la porte chinoise" a découvert "le premier art à deux dimensions que regardent sans dédain, des artistes européens: le premier art allusif et non illusionniste, qu'ils tiennent pour convaincant et non pour maladroit". " Comme Baudelaire a été l'un des premiers critiques à attirer l'attention sur le caractère prémédité de la composition de l'œuvre d'art, en écrivant notamment dans le Salon de 1846 qu'un "tableau est une machine dont tous les systèmes sont intelligibles pour un œil exercé, où tout a sa raison d'être [...]", "Malraux peut alors lui prêter la formule de Maurice Denis et écrire que "Baudelaire est sans doute le premier écrivain pour qui la peinture puisse être formes et couleurs en un certain ordre assemblées". " La majoration du fait pictural par Baudelaire lui a permis de saisir l'importance de la peinture de Manet.

En effet, vis-à-vis de l'œuvre de Manet, la position de Baudelaire contraste avec celle de la plupart des critiques de l'époque. Malraux cite quelques unes des réflexions les plus féroces que l'on a faites au sujet de l'Olympia (1863). "Enseigne de la femme à barbe...", "femme verte peinte avec des pinceaux à vaisselle...", "Sorte de gorille femelle...", tel est le ton de la critique. ${ }^{18}$ Même Théophile Gautier, ${ }^{19}$ grand poète et grand critique d'art et peintre lui-même, n'a pas saisi le sens de la démarche de Manet et a condamné sans appel son œuvre comme en témoigne ce jugement que cite Ma-

${ }^{18}$ (L'Intemporel, Op. cit.: 37.) Malraux ne précise pas le nom de ces critiques malveillants, ni le journal où été publiés leurs articles. Ces propos révèlent à ses yeux un état d'esprit général qui dépasse ces critiques. Par contre Georges Bataille, dans son Manet, fournit toutes les informations sur les critiques et les journaux qui ont participé au "scandale de l'Olympia”. Il nomme en particulier un certain Amédée Cantaloube, auteur de "la gorille femelle", et la fille de Théophile Gautier. (Cf. BATAILLE, Georges Manet. Genève: Albert Skira, 1955: 49.)

19 "Théophile Gautier était préparé à son métier de critique d'art par sa formation de rapin. A dix-huit ans, il a fréquenté l'atelier du peintre Rioult. Membre du Cénacle, il a rencontré chez Victor Hugo de nombreux artistes et approché Victor Hugo", écrit P. G. Castex dans Baudelaire, critique d'art. Paris: SEDES, 1969: 14. Théoricien des "transpositions d'art", Gautier a transcrit des tableaux de Zurbaran et de Ribera dans son recueil Espanâ (1845). 
lraux: "M. Manet, écrit Théophile Gautier, a cet honneur d'être un danger. Le danger est passé maintenant; Olympia ne s'explique d'aucun point de vue, même en la prenant pour ce qu'elle est, un chétif modèle étendu sur un drap." Un admirateur de Manet comme Zola se trompe lui aussi sur la signification de cette œuvre qu'il défend au non du réalisme. ${ }^{20} C^{\prime}$ est sans doute à lui que songe Malraux quand il écrit: "On parle de réalisme, puisqu'on ne peut parler d'idéalisation; mais adversaires et admirateurs sentent confusément que le réalisme n'est pas plus en cause que la caricature, la parodie ou le romantisme de la laideur."*

L'approche de Baudelaire differe beaucoup de la réaction hostile de Théophile Gautier et de la lecture erronée de Zola. Baudelaire, comme l'a remarquablement souligné P. George Castex, a été très proche de Manet, et a exercé sur lui une grande influence. ${ }^{21}$ Du temps de l'Olympia, il disait de lui: "Je suis un des premiers qui l'ont compris."* Mais s'il ne lui a pas consacré une grande étude comme il l'a fait pour Delacroix et Constantin Guys, c'est d'une part parce qu'il était déjà en cette année 1865 malade et affaibli, ' et d'autre part parce que Manet allait plus loin que lui, mettait en question la conception que la poète s'était faite de la peinture: "Car Manet est aussi éloigné que possible de Delacroix, l'idole de Baudelaire. Manet n'est pas un

\footnotetext{
${ }^{20}$ Dans un article paru le 1er janvier 1867 dans le Revue du XIXe siècle sous le titre "Une nouvelle manière en peinture: Edouard Manet", Zola défend l'artiste au nom de la vérité et du réalisme. Évoquant Olympia, il écrit: "Le corps lui-même de l'enfant a des paleurs charmantes; c'est une jeune fille de seize ans, sans doute un modèle qu'Edouard Manet a tranquillement copié tel qu'il était [...]. A la différence des peintres du XVe. siècle qui corrigent la nature et mentent, Edouard Manet, poursuit Zola, s'est demandé pourquoi mentir, pourquoi ne pas dire la vérité: il nous a fait connaitre Olympia, cette fille que vous rencontrez sur les trottoirs et qui serre ses maigres épaules dans un mince châle”. (Le Bon combat. Paris: Hermann, 1974: 89.) Zola fait ainsi d'Olympia une sœur de ses héroïnes chétives et fanées. Manet lui apparait même comme un "artiste moderne, un réaliste, un positiviste" (Idem: 165.) C’est pour cela que Gaëtan Picon a pu écrire dans la préface de cet ouvrage que "Zola ne s'est pas mieux compris qu'il n'a compris Manet" (Idem: 22).

21 "Baudelaire et Manet ont entretenu, à partir de 1858, d'excellentes relations. Manet subit l'influence de Baudelaire. La tradition rapporte que le poète lui aurait suggéré le sujet de son Buveur d'absinthe, ainsi que la négresse et le chat noir d'Olympia. C'est aussi sur les conseils de Baudelaire que Manet peignit en 1862 son grand tableau La Musique aux Tuileries, où Baudelaire figure à gauche, parmi d'autres contemporains. On doit encore à Manet l'unique portrait de Jeanne Duval qui appartient au musée de Budapest, ainsi qu'un portrait gravé, reproduit dans la biographie de Baudelaire par Asselineau [...] en 1862. Baudelaire composait un quatrain sur Lola de Valence, et faisait de lui un bel éloge au début de l'article 'Peintres et aquafortistes', écrit P. Georges Castex dans Baudelaire critique d'art, op. cit., 1969: 75.
}

* (L'Intemporel, op. cit.: 38.)

(Idem: 42.)

* (Idem, ibidem.)

* (Voir BATAILLE, Georges. Manet. Paris: Albert Skira, 1994: 33.) 
génie tourmenté, il ne cherche pas à exprimer sur ses toiles le drame humain, il ne pense pas et se contente de voir. Le sujet ne compte guère pour lui, et ne lui fournit qu'un prétexte pour peindre", "écrit P. Georges Castex. La lettre que Baudelaire écrit à Manet en 1865 pour le réconforter de l'accueil hostile que le public et la critique ont fait à l'Olympia contient une formule paradoxale qui a été jugée comme une condamnation de l'entreprise du peintre: "Vous n'êtes que le premier dans la décrépitude de votre art." ${ }^{\text {"* }}$. Georges Castex y lit une "restriction terrible et injuste"* et Gaëtan Picon, "le refus [...] de l'art ainsi annoncé", 22 mais Georges Bataille y perçoit un hommage: "autant dire qu'il l'aima sans savoir ce que voulait dire cette vigueur 'individuelle' qui sortait de la tradition." ${ }^{\prime 3}$

Malraux, sans aller totalement dans le sens de Bataille, propose une analyse plus nuancée. Dans La Tête d'obsidienne, il reprend cette citation dans un contexte précis et en donne une interprétation moins négative que ne le croit Claude Pichois. ${ }^{24}$ Notons d'abord que Malraux altère légèrement la formule de Baudelaire: "Vous n'êtes que le premier dans la décrépitude de votre art" devient: "N'oubliez pas que vous n'êtes que le premier dans la décadence de votre art."* Pour Malraux, ce jugement exprime la "position troublante" de Baudelaire qui a su deviner dans l'art de Manet un tournant décisif dans l'histoire de la peinture, dans la mesure où l'artiste s'est inscrit en faux contre le primat du sujet, contre le technique du modelé, de la perspective, du clair-obscur et contre toute expression psychologique. Avec lui, on assiste à la fin de l'Irréel, à la tombée en décrépitude de l'esthétique néo-classique et romantique que Manet n'exploite plus que comme des formes sans contenu: "L'Exécution de Maximilien [...] c'est le Trois Mai de Goya moins ce que ce tableau signifie", ${ }^{25}$ écrit Malraux.

Baudelaire aurait ainsi perçu dans la peinture de Manet la nouvelle aventure de l'art moderne, mais il ne pouvait pas la par-

22 PICON, Gaëtan. 1863: Naissance de la peinture moderne. Genève: Albert Skira, 1974, p. 25.

${ }^{23}$ Georges Bataille nuance cependant son jugement en écrivant: "Il lui échappa que Manet, justement de cette décrépitude, en brisant avec l'ordre du passé, tirait une contrepartie positive [...]" (Manet, op. cit.: 33.)

${ }^{24}$ Claude Pichois ne tient compte que de la phrase finale de l'analyse et estime qu'elle donne le "simple et véridique commentaire que voici: "La peinture que [Baudelaire] oppose à Manet, c'est celle de Delacroix" ("Malraux et Baudelaire" Europe $n^{\circ} 727 / 728$, nov-déc 1989: 173.)

${ }^{25}$ (Le Musée imaginaire, op. cit.: 42.) Cette phrase est citée et commentée par Georges Bataille dans son (Manet, Op. cit.: 35.) 
tager, parce que son esthétique et son "spiritualisme" appartiennent à l'ère de l'Irréel sur laquelle a régné de Delacroix.

Le fait d'avoir entrevu ce qui se joue dans la peinture de Manet comme mise en cause de l'héritage culturel, est interprété par Malraux comme une attitude qui prédispose Baudelaire à saisir la signification de l'art moderne et à apprécier l'invasion du musée imaginaire par les arts sacrés: "Il entendrait vite le langage des masques, des fétiches de Miro." Ainsi, de la même manière que "Baudelaire, qui n'était pas musicien, a deviné Wagner”, ${ }^{26}$ il a pressenti, aux yeux de Malraux, la nouvelle aventure de l'art qui commence avec Manet.

"Pour que Manet puisse peindre le Portrait de Clemenceau, il faut qu'il ait résolu d'oser y être tout, et Clemenceau, presque rien”, écrit Malraux dans Les Voix du silence. ${ }^{*}$ Ainsi l'art du peintre cesse d'être un ensemble de moyens au service d'une fiction pour devenir "un langage indépendant des choses représentées, aussi particulier que celui de la musique". ' Ce même mouvement d'émancipation, Malraux l'avait déjà reconnu dans la poésie de Baudelaire qui récuse l'anecdote au profit d'une harmonie suggestive: "La même grande aventure transformait la poésie, et de la même façon: avec Baudelaire, elle cessait de raconter." 'Limage qu'utilise Baudelaire pour évoquer le tableau de Manet, Lola de Valence (1862), le fameux "bijou rose et noir", ${ }^{27}$ est interprétée par Malraux au sens chromatique du terme. Elle désigne cet accord nouveau que recherchent aussi bien le peintre que le poète: "Lola de Valence n'est pas tout à fait 'un bijou rose et noir', mais Olympia commence à l'être, et dans la Pendule de marbre de Cézanne, la pendule sera réellement noire, le grand coquillage réellement rose". 'L'auteur associe encore davantage le poète au peintre en commentant le goût de Manet pour l'esquisse qui met en valeur la matière picturale, avec les mots mêmes que Baudelaire

${ }^{26}$ (Le Miroir des limbes, op. cit.: 745.) Malraux fait allusion à l'article publié par Baudelaire sous le titre "Richard Wagner et Tannhaïser à Paris" dans la Revue Européenne, du 1er avril 1861. (Cf. Curiosités esthétiques, op. cit.: 689-728.)

${ }^{27}$ Il s'agit du célèbre quatrain intitulé "Lola de Valence» qui fait partie de la section "Epigraphes" des Fleurs du Mal, Poème n XV. La "Note de l'éditeur" précise que "ces vers ont été composés pour servir d'inscription à un merveilleux portrait de Mademoiselle Lola, ballerine espagnole par M. Edouard Manet qui comme tous les tableaux du même peintre a fait esclandre. La muse de M. Charles Baudelaire est si généralement suspecte qu'il s'est trouvé des critiques d'estaminet pour dénicher un sens obscène dans le bijou rose et noir. Nous croyons, nous, que le poète a voulu simplement dire qu'une beauté d'un caractère à la fois ténébreux et folâtre, faisant rêver à l'association du rose et du noir" (BAUDELAIRE, Charles. Les Fleurs du mal. Paris: Garnier - Flammarion, 1964: 181).
* (La Tête d'obsidienne, op. cit.: 204.)

(Op. cit.: 99.)

(Idem: 110.)

(Idem, ibidem.)

* (Idem: 102.) 
a trouvés pour désigner ce procédé: "Ce qu’on appelait le faire dans le langage d'atelier, y prend la place du 'rendu'."

Mais ce qui unit le plus profondément l'approche esthétique de Malraux à celle de Baudelaire, c'est la primauté que l'un et l'autre accordent à la poésie.

En effet, l'exploration malrucienne de l'art laisse sur son parcours un cortège d'images et de rythmes qui redoublent le poème plastique d'un discours poétique tour à tour lyrique, épique ou tragique, car l'effet de l'art ne peut être dit que par un nouvel acte de création. Baudelaire dont Malraux n’a cessé de se réclamer n’a-t-il pas écrit que: "[...] le meilleur compte rendu d'un tableau pourra être un sonnet ou une élégie"? Et donnant lui-même, l'exemple, il a composé "Les Phares" et la série des "Epigraphes" où il évoque l'œuvre de Daumier, de Manet et de Delacroix.

Présentation d'un fragment inédit

Baudelaire

[Au-delà du malheur, mais pas comme le bonheur]

Baudelaire, malade, traqué, désespéré, regarde le dernier reflet du jour sur la face de sa hargneuse quarteronne, mais s'il fait à son tour monter du fond du temps le chuchotement sacré "Mère des souvenirs, maîtresse des maîtresses...”, la nuit qui descend sur ces deux visages flétris ensevelit doucement le mensonge de la terre. Jeanne Duval cesse d'être Jeanne Duval: sous la lueur mourante des tisons qui rougeoient pour les siècles, l'invincible amour rejette à l'apparence les ombres de l'éphémère, recompose le monde selon sa vérité. Baudelaire écrit-il le Balcon pour s'exprimer, ou s'exprime-t-il pour tenter d'atteindre ce qui n'est pas seulement la transcendance de l'amour, mais la destruction de l'irrespirable apparence, de la loi des choses que gouverne pour lui l'irrémédiable - d'atteindre la poésie? Il est faux que l'œuvre délivre l'artiste de l'angoisse, mais il est vrai que si cette ouvre est ce qu'il en attend, elle le reliera fugitivement au monde qui est au-delà de l'angoisse - au-delà du malheur, non comme le bonheur, mais comme l'absolu est au-delà de l'impermanence de l'Inde...* 


\section{Moncef Khémiri}

Professor da Universidade de La Manouba, em Tunis, e autor de Malraux écrivain d'art (Hermann, 2006). Publica regularmente artigos sobre os escritos estéticos de André Malraux na Revue des Lettres Modernes et na Revue André Malraux Review.

\section{Resumo}

Este ensaio tem por objetivo a comparação do pensamento estético de Baudelaire com o de Malraux, a partir do estudo inaugural de Claude Pichois sobre o lugar de destaque que ocupa o autor de Les Fleurs du mal e das Curiosités esthétiques na reflexão de André Malraux. Mas não só: ele acrescenta às fontes já conhecidas a correspondência de Malraux, sua "biblioteca estética" e os dossiês da gênese de La Métamorphose des dieux que o autor deste artigo teve a oportunidade de consultar para a edição dos Escritos sobre a arte de André Malraux na edição da Pléiade.

\section{Abstract}

The aim of this essay is to compare Bauderaire's views on aesthetics with those of Malraux, having as a starting point Claude Pichois' pioneer study on the important place occupied by the author of Fleurs du mal and $\mathrm{Cu}$ riosités esthétiques in the thoughts of André Malraux. But not only that: it adds Malraux's correspondence to the sources already known: his "aesthetic library" and the papers concerning the genesis of La Metamorphose des dieux that the author of this article had the opportunity to research for the Pléiade edition of André Malraux's Écrits sur l'art.

\section{Résumé}

Cet essai a pour but de comparer la pensée esthétique de Baudelaire et celle de Malraux, à partir de l'étude inaugurale de Claude Pichois sur la place importante qu'occupe l'auteur des Fleurs du mal et des Curiosités esthétiques dans la réflexion d'André Malraux. Mais non seulement: il ajoute aux sources déjà connues la correspondance de Malraux, sa "bibliothèque esthétique" et les dossiers de genèse de La Métamorphose des dieux que l'auteur de cet article a eu l'occasion de consulter pour l'édition des Ecrits sur l'art d'André Malraux en Pléiade.
Palavras-chave: Malraux Baudelaire; crítica estética; crítica genética.

Key words: Malraux; Baudelaire; aesthetic criticism; genetic criticism.

Mots-clés: Malraux; Baudelaire; critique esthétique; critique génétique.

Recebido em 05/02/2008

Aprovado em 14/04/2008 